\title{
Research on Decision Evaluation Model of HOV Lane Setting
}

\author{
Ding Lv $\mathbb{D}^{1,2,4}$ Qunqi Wu, ${ }^{1,4}$ Bo Chen, ${ }^{3,4}$ and Yahong Jiang ${ }^{3,4}$ \\ ${ }^{1}$ School of Economics and Management, Chang'an University, Xi'an 710064, China \\ ${ }^{2}$ Research Center for Urban Public Security, Shaanxi Police College, Xi'an 710021, China \\ ${ }^{3}$ College of Transportation Engineering, Chang'an University, Xi'an 710064, China \\ ${ }^{4}$ Center of Comprehensive Transportation Economic Management, Chang'an University, Xi'an 710064, China \\ Correspondence should be addressed to Ding Lv; lvding@chd.edu.cn
}

Received 18 September 2021; Accepted 20 October 2021; Published 28 November 2021

Academic Editor: Bai Yuan Ding

Copyright (C) 2021 Ding Lv et al. This is an open access article distributed under the Creative Commons Attribution License, which permits unrestricted use, distribution, and reproduction in any medium, provided the original work is properly cited.

\begin{abstract}
In order to achieve the purpose of improving the travel efficiency of commuters in the periphery of the city, expanding the beneficiary groups of urban rail transit, and alleviating urban road traffic congestion, when planning and setting up HOV in the periphery of the city, it is necessary to analyze the feasibility of HOV lane setting from both the demand conditions and the setting conditions. This paper combines machine learning to construct a decision-making evaluation model for HOV lane setting and studies the optimal layout model and algorithm of HOV lanes in service rail transit commuter chain. The setting, planning, and layout of HOV lanes are a two-way interactive process of traveler's path selection and designer's road planning. Finally, after the model is constructed, the performance of the system model is verified. The results show that the system studied in this paper can be used for traffic data and lane planning analysis. Therefore, in the process of urban operation, the HOV model constructed in this paper is mainly used to alleviate urban traffic and improve urban operation efficiency.
\end{abstract}

\section{Introduction}

In order to ensure the smoothness of urban traffic and the sustainable development of traffic, since the late 1990s, major cities in China have undertaken a series of actions such as road rectification, scale expansion, and construction of transportation infrastructure to improve the current state of traffic congestion. However, the construction speed is far behind the growth rate of the traffic volume in the transportation system, and the current total road scale and traffic management level are far from the actual needs of travelers in the transportation system. Moreover, the growth rate of transportation demand is far greater than the rate of new infrastructure construction. In addition, actual experience over the years has shown that, relying only on new roads, increased investment and other methods to deal with traffic congestion will not solve the problem and will even plunge the supply of road resources into a vicious circle, which will worsen the imbalance between supply and demand. In particular, when the road network structure and basic framework of medium and large cities have been determined, the available land resources are already very limited, and blind expansion can only further squeeze the space resources of the city. However, with the increasing demand for travel, newly built roads will be filled with huge traffic, causing new traffic jams. This will further deepen the imbalance between supply and demand in the transportation system, make the contradiction between the supply and demand of road resources increasingly acute, and intensify the congestion problem. Because of the above phenomenon, a series of problems may arise, such as lower and lower travel efficiency of residents and more and more serious urban environmental pollution. Improving road utilization may become a more operational and effective method for alleviating traffic congestion in my country's big cities, and it is also a research direction with a lot of room for development in the future [1].

The setting of HOV lanes is an effective way to alleviate congestion. HOV is a dedicated lane with high loading rate. It was formally proposed in the United States as a concept in traffic demand management in the 1990s. As the name implies, the HOV dedicated lane refers to a management 
lane dedicated to vehicles with high load-bearing rates. The proposal of this concept provides a reasonable new idea for alleviating urban traffic congestion [2].

For improving urban traffic conditions, commuters are the main participants in the morning and evening peaks [3]. To study the optimal layout of HOV lanes in the service rail transit commuter chain, the route with the least total travel time cost can be selected from multiple road sections in the periphery of the city as the route for setting HOV lanes. Moreover, it can guide more commuters to travel by carpooling, then transfer to rail transit, and finally connect to other modes of travel to complete the commuting process.

This paper creatively establishes the decision-making evaluation model of high-performance vehicle lane setting combined with machine learning. The optimal layout model and algorithm of high-performance vehicle lanes in service rail transit commuter chain are studied. The setting, planning, and layout of HOV lane are a two-way interactive process of traveler's path selection and designer's road planning. Finally, after establishing the model, the performance of the system model is verified. The results show that the high-performance vehicle lane path optimization model constructed in this paper has a certain effect.

This paper is divided into five parts. The first two parts introduce the theory of rail transit commuter chain and HOV lane and then determine the research method of the attraction range of rail transit stations around the city. The third part of this paper establishes the decision-making evaluation model of high-performance vehicle lane setting combined with machine learning. The fourth part studies the optimal layout model and algorithm of high-performance vehicle lane in the service-oriented rail transit commuter chain. The setting, planning, and layout of HOV lane are a two-way interactive process of traveler's path selection and designer's road planning. Finally, after establishing the model, the performance of the system model is verified.

\section{Related Work}

Gadawe et al. [4] comprehensively summarized the development status of existing HOV lanes abroad. This paper sorted out the whole process of HOV lane development in chronological order, introduced the concept, function, type, and existing problems of HOV lane in turn, and also briefly described the doubts raised by scholars in other fields regarding this new thing. Moreover, the paper finally boldly predicted the research direction and development trend of HOV lanes in the next few years and elaborated in detail objectively and comprehensively the irreplaceability of $\mathrm{HOV}$ lanes and the inevitability of putting them into practical use.

Malipatil et al. [5] used a multimethod selection logit model to discuss the construction and application of $\mathrm{HOV}$ lanes in urban traffic systems. Starting from the current low utilization rate of bus dedicated lines, Rehman et al. [6] proposed a targeted approach to construct HOV lanes using idle or underutilized bus dedicated lines. Moreover, they used the existing data in the actual road network to perform inference calculations and conducted reasonable analysis in both the necessity and feasibility dimensions. Hamarashid et al. [7] selected a specific road section to analyze data and perform calculations and predicted and analyzed the final results. This type of research provides the necessary theoretical basis for introducing HOV lanes into China's actual road network for application. Matthews et al. analyzed traffic problems from the perspective of macro-coordination, and based on the comprehensive consideration of foreign transportation systems, they first proposed the concept of carpooling priority. Sivanathan et al. [8] introduced the management of HOV lanes into urban expressways and used the MNL model and analytic hierarchy process to explain in detail the necessity and possibility of prioritizing the development of HOV lanes under the current development situation. Koresh et al [9] conceived the combined construction of HOV lanes and BRT lanes, which, to some extent, broke our country's stereotype of thinking about HOV lane construction. Ning et al. [10] discussed the actual effect of HOV lanes in alleviating traffic congestion and analyzed the feasibility of setting HOV lanes in cities. This paper uses simulation and comparative analysis. Qiu et al. [11] used the traffic efficiency model as a tool to specifically analyze the functionality of HOV lanes in urban settings. Moreover, based on the current situation and forecasted traffic volume, it used the calculation and comparative analysis of traffic efficiency to analyze whether the construction of HOV lanes is feasible and proposed the decision-making basis for judgment when setting up HOV lanes. Liu et al. [12] analyzed the setting conditions of HOV lanes in actual operation, proposed $\mathrm{HOV}$ lane management methods that meet the actual situation, and designed four different types of cross-sections for HOV lanes according to different types. Sharif et al. [13] used VISSIM software to carry out a simulation comparison and analysis on the road conditions before and after setting HOV lanes and explained in detail the respective advantages and disadvantages and applicable conditions of HOV lanes and bus lanes. Arulselvi et al. [14] put forward the design and construction plan for the design of HOV lanes in more detail. Moreover, on the basis of on-site traffic investigation and data collection and sorting, they carefully considered the specific organic components of the road and gave a complete and specific HOV dedicated lane design plan. Finally, it used simulation software to evaluate the design. Li et al. [15] incorporated carpooling as an independent travel mode into the transportation system and introduced the two-level programming model into the problem of HOV lane construction to solve the optimal HOV lane layout. Raskar et al. [16] adjusted the relationship between the difference of the total utility and the planned HOV route from the perspective of whether the total utility of travel can be reduced and deeply studied the method of optimal design of the HOV dedicated lane. Sumi et al. [17] proposed a design method for $\mathrm{HOV}$ dedicated lanes that can improve road operation efficiency. For the first time in this paper, the idea of directly converting ordinary lanes into HOV lanes is proposed.

Although Adekitan et al. [18] has evaluated the operating performance of high-carrying lanes, most studies focus on the limited data obtained from one or a small number of road sections. The methods used in these studies may not be 
easily applied to larger studies. Some of them neglect largescale data processing, because it takes too much time. Moreover, other studies are only to illustrate the running performance of the short-circuit section of the high-carrying lane. For example, only the distance between vehicle detection stations (VDS) is considered in space. In addition, the conclusions drawn in the research of some specific road sections may be restricted by relevant conditions, such as the traffic demand and lane configuration of the road section. Therefore, it is difficult to extend the conclusions obtained to other suitable situations. By analyzing the fiveyear traffic accident data in the Traffic Accident Monitoring and Analysis System (TASAS) database, Li et al. [19] evaluated the safety performance of continuous and restricted high-carrying lanes. Among them, an interesting finding is that, in terms of accident rate and severity, restricted-access high-load lanes cannot provide better safety performance than continuous high-load lanes. Some studies compare the accident characteristics of two different types of high-carrying lanes, such as accident location, accident type, and frequency. Bellucci et al. [20] pointed out that, in terms of safety, the partition design of the space separation wall is better than the partition design of the buffer zone, at least better than the continuous access design. Wang et al. [21] pointed out that the accident rate of continuous high-carrying lanes is lower than that of restricted high-carrying lanes. However, the continuing impact after the accident has not been studied in depth. As a nonrepeatable cause of congestion, traffic accidents are an important part of the Freeway Traffic Management System (FTMS). Many accident analysis methods focus on the analysis of conventional highways. Traffic accidents on high-load lanes will affect the overall performance of the high-load lanes, and their accident research and analysis have great significance.

\section{Decision Evaluation Algorithm for HOV Lane Setting}

It can be found from Figure 1 that this is a process problem with many stages, and each stage has many decision-making processes. In order to solve this type of problem, a method is proposed, namely, the dynamic programming method. Therefore, the research direction of the dynamic programming method is: how to select the optimal series of decisions from a multistage and multidecision process? After the decision-making system process with time as a variable, the decision of each stage of the system is obtained according to the current state of the system. At the same time, the obtained decision immediately causes the current state to transition to the next moment state, and the next moment state is selected by the optimization principle to get the next moment decision, and at the same time, the next moment state is generated. In this way, the state of the system is constantly moving, and decisions are constantly being produced. Therefore, a series of decisions composed of one optimal decision is produced, which gives the impression that it is a process of continuous movement. Therefore, it is called a dynamic programming method.

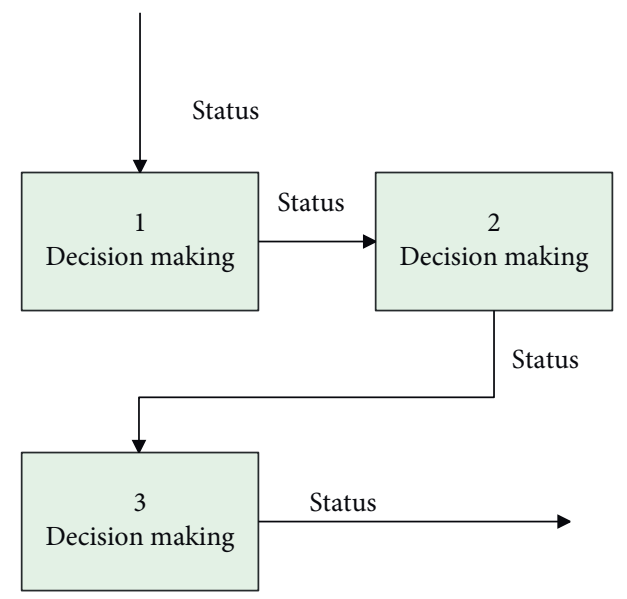

FIgURE 1: Schematic diagram of multistage and multidecision process.

The state that the system transitions from the current state to the next moment is related to the system function. People generally call the mathematical model of the state transition of the system as the state transition equation, which is an important part of the dynamic programming method [22]:

$$
x(k+1)=F(x(k), u(k), k), \quad k=0,1, \ldots, N-1 .
$$

Among them, the function $F(\cdot)$ represents the function of the system, the character $k$ represents the $k$-th stage in a system with $\mathrm{N}$ stages, the state $x(k)$ represents the state variable of the decision-making system in the $k$-th stage, and the decision $u(k)$ represents the action strategy taken by the system process when the state is $x(k)$.

An important member of the dynamic programming method is the efficiency function $U[x(k), u(k), k]$. The socalled efficiency function refers to the benefit value $U(\cdot)$ obtained by the system when the system state $x(k)$ is transferred to the state $x(k+1)$ at the next moment of the system process because of the decision $u(k)$ in the $k$-th stage of the system process.

$$
J(x(i), i)=\sum_{k=1}^{N} \gamma^{k-i} U[x(k), u(k), k], \quad k=1,2, \ldots, N .
$$

In the above formula, $\gamma$ represents the discount factor, and its value range is $0<\gamma \leq 1 . J(x(i), i)$ represents the sum of the instant benefits obtained from the initial time $i$ and the initial state $x(i)$ when the system $q$ executes the system decision-making process until the end of the entire system decision-making process [23].

However, in dynamic programming, $J(x(i), i)$ is not the index that people want. What people care about is its optimal index, which is the optimal performance index function:

$$
J^{*}(x(i))=\underset{\{u(k), \ldots, u(N)\}}{o p t} \sum_{k=i}^{N} \gamma^{k-i} U[x(k), u(k), k] .
$$


In the formula, $J^{*}(\cdot)$ represents the performance index function obtained when the decision of each stage of the process system is optimal. opt stands for maximum or minimum.

To understand the optimal performance index function, it is necessary for us to understand the principle of optimization. Its theory is as follows:

We assume that there is a set $\Psi$, which has many elements and many subsets. When $\Phi$ is a subset of $\Psi$, the largest element $\mu$ in set $\Psi$ is the largest element in set $\Phi$. When the subset $\Phi$ contains the largest element in the set $\Psi$, then the largest element $\varepsilon$ in the set $\Phi$ is the largest element in the set $\Psi[24]$.

In the 1950s, in order to solve the problem of multistage and multidecision system, Bellman proposed the core theory of dynamic programming method, namely, Bellman equation, according to the optimization principle. Its specific theory is as follows:

In a process system with $\mathrm{N}$ stages and $\mathrm{N}$ decisions, the optimal strategy sequence of the system is $u(0), \ldots, u(k), \ldots, u(N-1)$. Then, when the initial state $x(k)$ and the initial strategy $u(k)$ are used as the starting point, the system decision sequence $u(k), \ldots, u(k+1), \ldots, u(N-1)$ of the remaining $N-k$ stages of the process system must still be the optimal strategy sequence of the remaining stage.

The mathematical model is as follows:

$$
J^{*}(x(k))=\min _{u(k)}\left\{U(x(k), u(k))+\gamma J^{*}(x(k+1))\right\} .
$$

The nonlinear discrete system can be regarded as a multistage decision-making system, so it is suitable to use the dynamic programming method. After years of exploration and research, the basic model of using dynamic programming in discrete systems is as follows:

First, the mathematical model of the state transition equation of the nonlinear discrete system to be solved at the $\mathrm{k}$-th stage is as follows:

$$
x(k+1)=F(x(k), u(k), k), \quad k=0,1, \ldots
$$

The conditions of the initial state are

$$
x(0)=x_{0} .
$$

For $x(0)$, the corresponding system performance index function when $x(N)$ is in domain $G(x(N), N)=0$ is

$$
J=G(x(N), N)+\sum_{k=1}^{N-1} U(x(k), u(k), k) .
$$

Based on the optimality principle of Bellman equation, after step-by-step sorting and simplification, the mathematical expression of the optimal performance index function is finally obtained as

$$
\begin{aligned}
J^{*}(x(k)) & =\min _{u(k)}\left[U(x(k), u(k))+J^{*}(x(k+1))\right], \\
k & =1,2, \ldots, N-1 .
\end{aligned}
$$

To solve this formula, we must also obtain the optimal performance index function of the last stage $x(N)$, namely $J^{*}(x(N))$.

$$
J^{*}(x(N))=\min _{u(N)}[G(x(N))+U(x(N), u(N))] .
$$

If the continuous time is regarded as composed of many very small time periods, then the continuous-time system becomes a discrete system. Therefore, continuous-time system models can also be solved using dynamic programming methods. The following is the mathematical model of dynamic programming method applied in continuous-time system. First, we assume that the mathematical model of the state transition equation in the continuoustime system is

$$
\dot{x}(t)=f(x(t), u(t), t), \quad t_{0} \leq t \leq t_{f} .
$$

The initial state of the system is

$$
x\left(t_{0}\right)=x_{0} .
$$

For $x\left(t_{0}\right)$, the corresponding system performance index function when $x\left(t_{f}\right)$ is in domain $G\left(x\left(t_{f}\right), t_{f}\right)=0$ is

$$
J=G\left(x\left(t_{f}, t_{f}\right)\right)+\int_{t_{0}}^{t_{f}} U(x(t), u(t), t) \mathrm{d} t .
$$

In the formula, the final moment state $x\left(t_{f}\right)$ of the continuous-time system is fixed, $x(t)$ and $u(t)$, respectively, represent the state vector and control vector of the continuous-time system at time $t$, the functions $G(\cdot)$ and $\int$ are continuously differentiable, and the function $U(\cdot)$ is the efficiency function and continuously differentiable. It represents the income of the continuous-time system at time $t$.

When the state vector of the system is $x(t)$, the corresponding control vector $u(t)$ can be found to make the performance index function optimal:

$$
J(x(t), t)=\min _{u(t)}\left\{G\left[x\left(t_{f}, t_{f}\right)\right]+\int_{t_{0}}^{t_{f}} U(x(t), u(t), t) \mathrm{d} t\right\} .
$$

Based on the Bellman equation, when the performance index function of the continuous-time system is taken as the minimum $u(t)$, the function equation is

$$
J(x(t), t)=\min _{u(t)}\left\{\int_{t}^{t+\Delta t} U(x(t), u(t), t) d t+J(x(t+\Delta t), t+\Delta t)\right\} .
$$


After a series of derivation and simplification operations, the above equation is finally simplified to obtain the Hamilton-Jacobi equation as follows:

$$
-\frac{\partial J^{*}(x(t), t)}{\partial t}=\min _{u(t)}\left\{U(x(t), u(t), t)+\left(\frac{\partial J^{*}(x(t), t)}{\partial x(t)}\right)^{T} f(x(t), u(t), t)\right\} .
$$

Its optimal performance indexes at the final moment are

$$
J^{*}\left(x\left(t_{f}, t_{f}\right)\right)=G\left[x\left(t_{f}, t_{f}\right)\right] .
$$

Based on the above two formulas, the solution of the continuous-time system can be solved.

Reinforcement learning is defined as a machine intelligence method that successfully combines dynamic programming and supervised learning to solve problems that neither of these two methods can solve alone. Supervised learning is a method of approaching the target through a lot of training, but its approach is not random, but determined by the tutor signal provided by the outside. That is to say, if there is no external tutor signal to supervise the learning, it is impossible to achieve success. Unfortunately, in people's real life, people have not found clear answers to many questions, so people cannot provide matching mentor signals.

In a standard reinforcement learning model, an agent (agent) interacts dynamically with its environment. These interactions are the forms that the agent uses to explore the surrounding environment and select a strategy through the results of the exploration. This strategy corresponds to an action of the agent. When the action is executed by the agent, it will have a certain impact on the environment. influences. This influence will notify the agent through some kind of enhanced signal, and the agent can judge whether the influence is good or bad based on the feedback signal. A typical reinforcement learning system consists of three basic parts: environment, reinforcement module, and value function.

(1) The environment of reinforcement learning can be understood as follows: the reinforcement learning system learns how to establish an optimal mapping to select the optimal action through error training that continuously interacts with the dynamic environment. This dynamic environment must be at least partially observable by the reinforcement learning system. It is said to be observation, but it is actually detected by various sensors and then described by some related symbols such as voltage and current, so that the agent can use it.

(2) The reinforcement module can be understood as follows: the reinforcement learning system learns a mapping from situation to action through error training that interacts with the dynamic environment. Therefore, the goal of reinforcement learning is defined by a concept called reinforcement function. The so-called reinforcement function is a function that can maximize the sum of the benefits of the agent in the future. After the reinforcement learning system performs an action according to a state, it will obtain a reinforcement signal as a reward or punishment. The purpose of the reinforcement learning system is to maximize the sum of all rewards obtained by performing actions from the beginning of the initial state to the end of the final state through learning.

One of the main tasks of reinforcement learning system designers is to define a reinforcement function, so that this function has a proper learning goal. Although the designer can freely define the reinforcement function of the reinforcement learning system, in order to achieve people's desired goals, the designer has some links when constructing the reinforcement function, for example, purely delayed rewards and punishments. When the system state is the final state, people hope that the reinforcement incentive of pure delayed reward function class is zero.

(3) Value function: how does the agent learn to choose those good actions? Or how to measure the effectiveness of an action? Therefore, the concept of value function is introduced into reinforcement learning. The so-called value function refers to the enhancement function in any state plus the maximum value of the sum of all enhancement functions from this state to the final state.

The schematic diagram of reinforcement learning is shown in Figure 2. The parameter module includes these parts: policy module $P$, enhancement module $R$, input module $I$, and the current state $s$ of the agent receiving environment. When the agent (consisting of the strategy module $P$, the reinforcement module $R$, and the input module $I$ ) interacts with the dynamic environment, the agent receives the current state $\mathrm{S}$ of the environment and becomes $i$ after processing $I$. After that, the strategy module derives strategy a based on the $r$ at the previous moment and the current $i$. After that, under the action of a, the dynamic environment $T$ changes its state from $s$ to $s^{\prime}$ and, at the same time, sends a return $r$ to the agent.

In fact, reinforcement learning is developed from the Markov process. In a Markov process environment, the agent selects an action a from the set of all actions, which makes the state of the environment system transfer and returns a reward and punishment value $r$ to the agent. Its mathematical expression is as follows: 


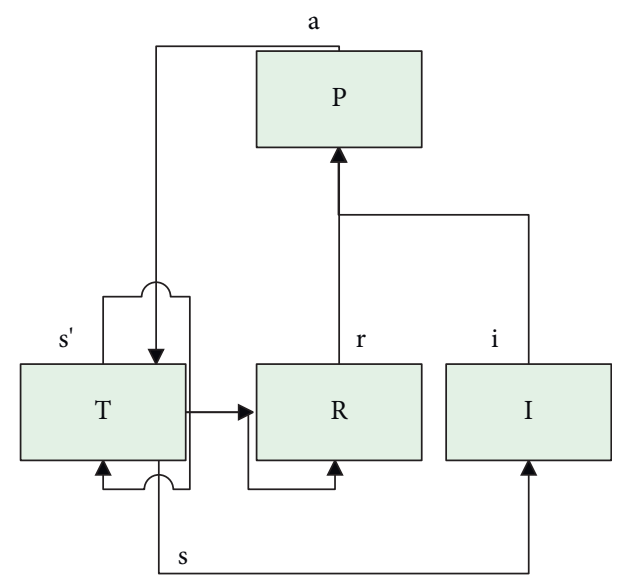

FIGURE 2: Schematic diagram of reinforcement learning.

$$
\operatorname{prob}\left(s=s^{\prime} \mid s, a\right)=P\left(s, a, s^{\prime}\right) .
$$

The utility function obtains the value when the state is $s$ under the action of the strategy, and the mathematical expression form is

$$
U(s)=r(s)+\gamma \max _{a} E\left(\sum_{s^{\prime}}^{s_{N}}\left(T\left(s, a, s^{\prime}\right), U\left(s^{\prime}\right)\right)\right) .
$$

In the formula, $s_{N}$ is the final state of the system, and $\gamma$ is the discount factor. When action a is the optimal action in the set of all actions, then $U(s)$ is also the optimal value function in state $s$.

From the composition of GDHP, we know that it is very complicated, and what is even more troublesome is that it must calculate the second derivative $\partial^{2} J(t) / \partial R(t) \partial W_{c}$. Although it is much more complicated than the previous two types, the results obtained are much better than expected. In 1997, Danil and Donald gave a detailed analysis and calculation module in their paper to make the second derivative $\partial^{2} J(t) / \partial R(t) \partial W_{c}$ easier to calculate. Its structure is shown in Figure 3. When the squared difference is the smallest, we can get the update formula for evaluating the weight of the network:

$$
\begin{aligned}
\Delta W_{c}= & -\eta_{h}[J(t)-\gamma J(t+1)-U(t)] \frac{\partial J(t)}{\partial W_{c}} \\
& -\eta_{d} \sum_{j=1}^{n} \frac{\partial^{2} J(t)}{\partial R_{j}(t) \partial w_{c}} E_{D} .
\end{aligned}
$$

Among them, $\eta_{h}, \eta_{d}$ are the learning rates of the execution network and the evaluation network, respectively, and $E_{D}$ is the squared difference of DHP.

In this figure, how does GDHP's evaluation network get $\partial^{2} J(t) / \partial R(t) \partial W_{c}$ ? First of all, we must be able to work smoothly in the GDHP design structure shown in Figure 4. Here, by using the output $J(t)$ and $\partial J(t) / \partial R(t)$ of the evaluation network, the burden of calculating $\partial^{2} J(t) / \partial R(t) \partial W_{c}$ will be minimized. In this way, the second

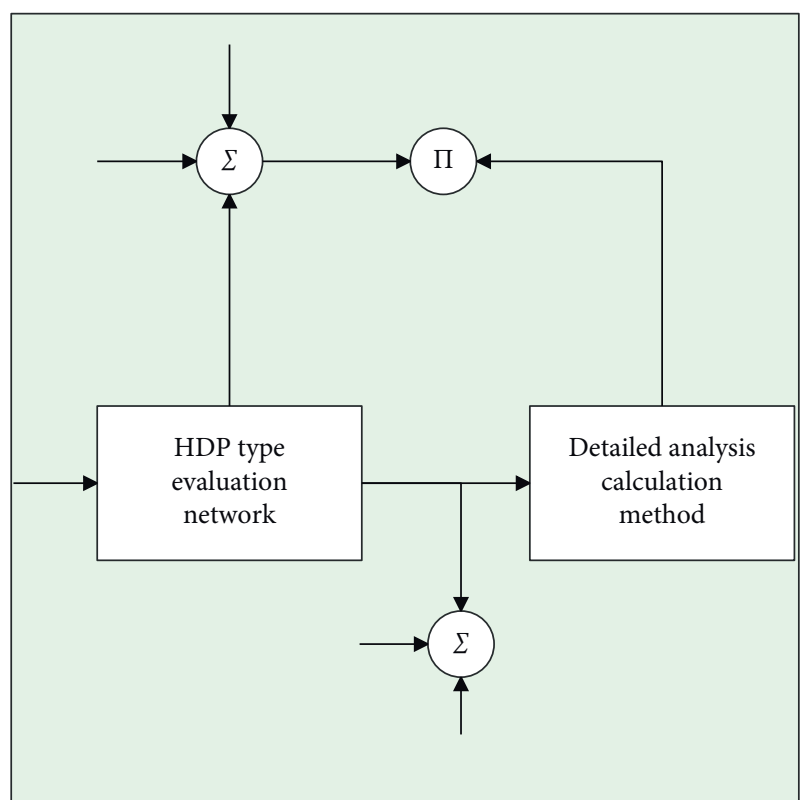

FIgURE 3: Schematic diagram of the structure of GDHP.

derivative $\partial^{2} J(t) / \partial R(t) \partial W_{c}$ can be obtained more conveniently by backpropagation.

The output $J(t)$ of the evaluation network does not return to the input $R(t)$ through the internal path to train the execution network, because if it is executed in this way, it will return to the working mode of HDP. We already have a high-quality method to obtain $\partial J(t) / \partial R(t)$, which is DHP. Therefore, DHP is put into GDHP as a part of GDHP.

A simple evaluation network is designed as shown in Figure 5:

From Figure 3, we know that its hidden layer includes two sigmoidal nerves and a linear output $J(t)$. The following equation can be obtained from the network:

$$
\begin{aligned}
J(t)= & f_{3} w_{35}+f_{4} w_{45}+R_{0} w_{05} \\
= & \frac{w_{35}}{\exp \left(-R_{0}(t) w_{05}-R_{1}(t) w_{13}-R_{2}(t) w_{23}\right)+1} \\
& +\frac{w_{45}}{\exp \left(-R_{0}(t) w_{04}-R_{1}(t) w_{14}-R_{2}(t) w_{24}\right)+1}+R_{0} w_{05} .
\end{aligned}
$$

To find the partial derivative $\partial J(t) / \partial R_{j}(t)$ of the input for the output, there are

$$
\begin{aligned}
\frac{\partial J(t)}{\partial R_{j}(t)} & =\sum_{3}^{4} f_{i}\left(1-f_{i}\right) w_{i 5} w_{j i}+w_{05} \delta_{j 0}(t), \\
i & =3,4 ; j=0,1,2 .
\end{aligned}
$$

Among them, $\delta_{j 0}(t)$ is the Kronecker function. Then, the partial derivative of the weight of the output layer and the weight of the hidden layer is calculated from the above formula, as shown in the following formula: 


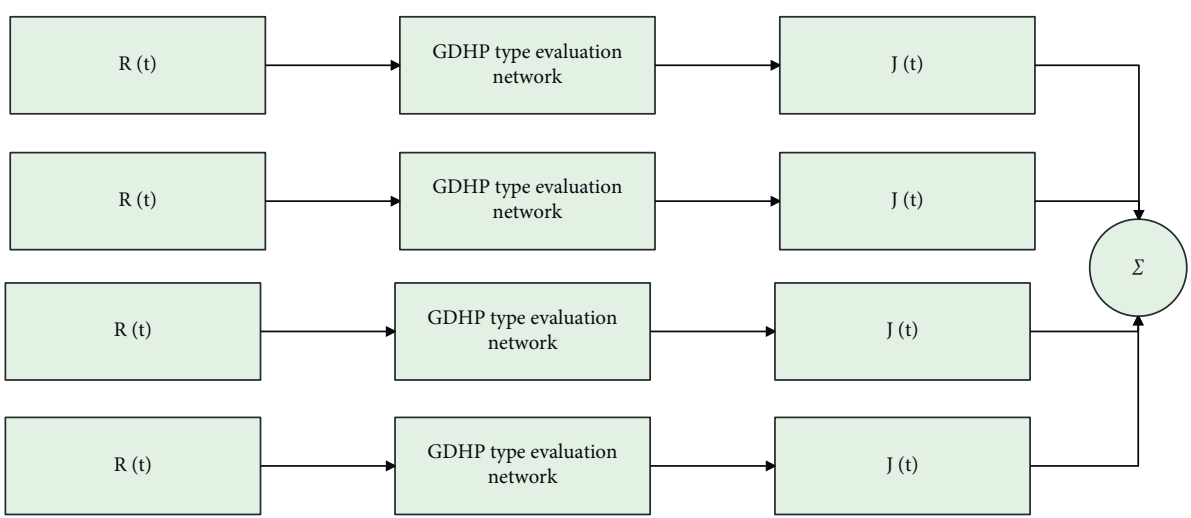

FIGURE 4: Evaluation network in a simple GDHP design.

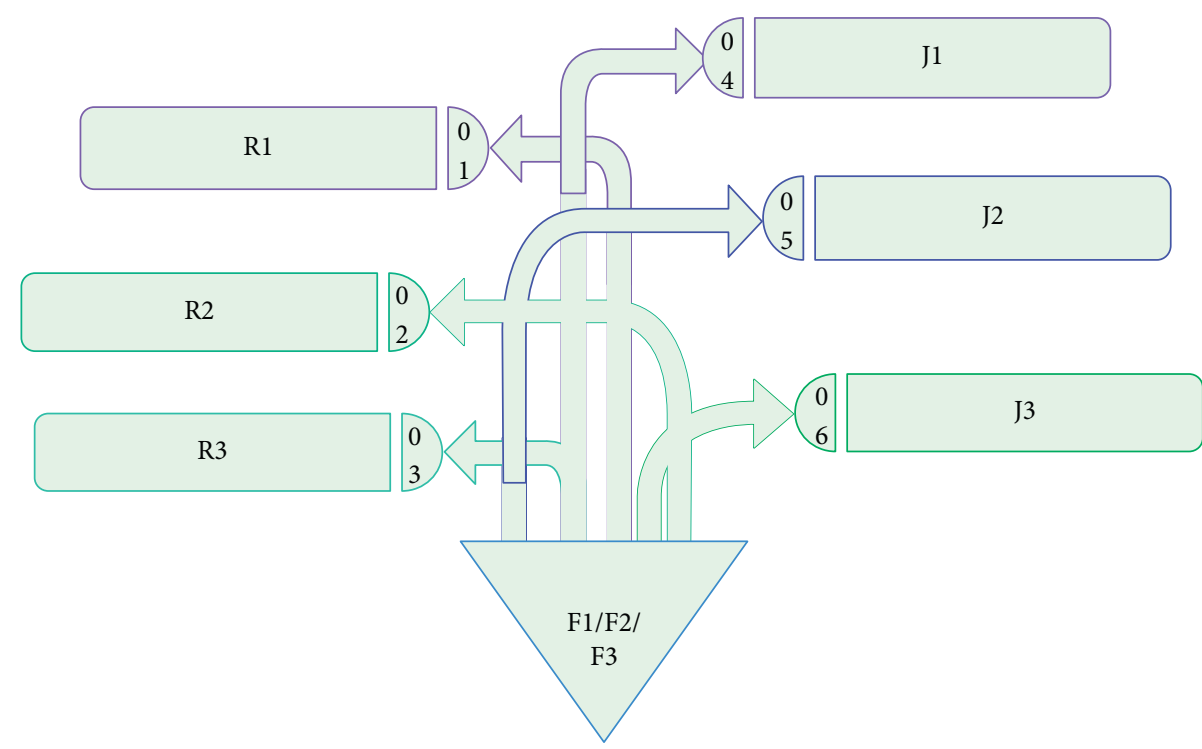

Figure 5: A simple network used to calculate an example of $\partial^{2} J(t) / \partial R(t) \partial W_{c}$ in the GDHP design.

$$
\begin{aligned}
\frac{\partial^{2} J(t)}{\partial R_{j}(t) \partial w_{i 5}} & =w_{j i} f_{i}\left(1-f_{i}\right), \quad i=3,4 ; j=0,1,2, \\
\frac{\partial^{2} J(t)}{\partial R_{j}(t) \partial w_{05}} & =\delta_{j 0}(t), \quad i=3,4 ; j=0,1,2, \\
\frac{\partial^{2} J(t)}{\partial R_{j}(t) \partial w_{j i}} & =f_{i}\left(1-f_{i}\right) w_{i 5}\left[1+\left(1-2 f_{i}\right) R_{j}(t) w_{j i}\right], \\
i & =3,4 ; j=0,1,2, \\
\frac{\partial^{2} J(t)}{\partial R_{j}(t) \partial w_{k i}} & =\left(1-2 f_{i}\right) R_{j}(t) w_{j i} f_{i}\left(1-f_{i}\right) w_{i 5,}, \\
i & =3,4 ; k=0,1,2 ; j=0,1,2, k \neq j .
\end{aligned}
$$

Then, we can use the basic formula to modify its form:

$$
\begin{aligned}
\Delta W_{j i}= & -\eta_{h}[J(t)-\gamma J(t+1)-U(t)] \frac{\partial J(t)}{\partial W_{j i}} \\
& -\eta_{d} \sum_{k=1}^{2} \frac{\partial^{2} J(t)}{\partial R_{k}(t) \partial w_{j i}}\left[\frac{\partial J(t)}{\partial R_{k}(t)}-\gamma \frac{\partial J(t+1)}{\partial R_{k}(t)}-\frac{\partial U(t)}{\partial R_{k}(t)}\right] .
\end{aligned}
$$

This is the weight update formula of GDHP.

\section{Decision Evaluation Algorithm for $\mathrm{HOV}$ Lane Setting}

Travelers are passengers who use it in the road network, and they are more concerned about the cost of travel. Therefore, the Stackelberg game problem is the essence of the optimal design of HOV lanes. That is, the planning department of the decision-maker and the follower traveler live in two levels of decision-making process, respectively, and they are relatively independent and affect each other. In order to achieve 
the purpose of maximizing their own interests, they are optimized separately, and finally the effect of the optimization of the HOV lane layout is achieved. This paper solves this problem by constructing a two-level programming model.

According to the actual operation of HOV lane, VSSM is selected as the simulation software, the micro simulation platform of HOV lane is built, and the evaluation index system of traffic flow characteristics and HOV Lane characteristics is established. In this paper, the effectiveness of the simulation platform is tested and corrected. Based on the current situation of HOV Lane setting, combined with the relevant standards and laws and regulations of foreign $\mathrm{HOV}$ system, this paper summarizes the setting conditions of domestic HOV lane and verifies the effectiveness of the scheme by comparing the evaluation results before and after.

The upper-level model starts from the perspective of the planner (i.e., the system). By deciding which road section to build HOV dedicated lanes, the system efficiency of the entire transportation network can be optimized. Therefore, by considering the performance indicators of the generalized cost of the selection system, the objective function of the upper model is formed. By optimizing the objective function, the optimal HOV channel set is found, and the optimization result ensures that the generalized cost of the entire road network system is minimized, and the efficiency is also optimal. The lower-level model starts from the standpoint of road network users (travelers) and considers travel users as the lower-level decision makers. By choosing the corresponding travel strategy under the road network conditions defined by the upper-level model, the lowest travel cost is achieved. The result of the traveler's choice will in turn affect the result of the HOV lane optimization design of the upper model.

The specific modeling steps and framework are shown in Figure 6. Passenger Car Flow Distribution includes all vehicles driving on regular roads.

The regional city structure diagram is shown in Figure 7.

The urban periphery studied in this paper refers to the urban fringe area, which includes inner and outer edges, and has the following characteristics: (1) it maintains close contact with the urban core area and is the main penetration zone of the core area; (2) its population grows rapidly, and its structure is complex; (3) its land properties continue to change, and the intensity of land development continues to increase; (4) it is an area with frequent commutes. Factors such as urban population density, land properties, and economic development will affect the determination of the extent of urban fringe areas. At the same time, economic, transportation, location, policy, and other factors will also affect the expansion of urban fringe areas. Therefore, according to the characteristics of different cities, the scope of the outer city should be determined specifically. The connection distance between the urban center and the periphery of the city in our country is usually $10-20 \mathrm{~km}$. The division of urban structure is not static. As the city's economic strength increases, and the scope of the urban core area increases, the original urban fringe area will gradually evolve into the core area, and the affected area will evolve into the fringe area. The development of the city is the process of continuously increasing the extent and scope of land use.

There is a dynamic relationship between transportation, economy, and land use. The convenience of transportation can drive the development of urban economy, the development of economy drives land use, and the use of land strengthens the development of transportation. The relationship between the three is shown in Figure 8 .

On the basis of the definition of the urban periphery and the commuter chain, we combine the research content of this article to summarize the definition of the rail transit commuter chain. That is, commuters in the periphery of the city use urban rail transit as the main travel mode at one time and connect with other modes of transportation to form a travel chain, as shown in the schematic diagram in Figure 9. This type of travel chain includes three processes: front-end travel (the travel phase of commuters starting from the residential area to the urban rail transit station), urban rail travel, and back-end travel (the commuter travels from the urban rail transit station to the work unit stage).

The rail transit commuter chain is divided into two types: simple and complex. The simple rail transit commuter chain refers to a chain that connects to the front and back ends of urban rail transit and has only one mode of transportation except walking. The complex rail transit commuter chain refers to a chain that connects the front and back ends of urban rail transit and has two or more modes of transportation except walking. In commuting travel, complex commuter chains usually bring a psychological burden to commuters, and frequent transfers cause time waste, so simple commuter chains are the first choice for commuters. Taking into account the limitations of the research conditions, the scope of this paper is defined as the simple rail transit commuter chain of "HOV-urban rail transitwalking".

Restricted by passenger demand and construction conditions, the characteristics of urban rail transit in the central area of the city and the outer area of the city are different. The urban rail transit in the central area is networked, and the urban rail transit in the outer city is often the end of a certain urban rail transit line, so determine the type of urban rail transit station outside the city as intermediate station or terminal station.

The urban rail transit in the periphery of the city is often the end of a certain urban rail transit line. Therefore, the type of urban rail transit station in the outer periphery of the city is determined as the intermediate station or the terminal station. When the distance between the urban rail transit station and the core area of the city is different, the radiation range is different. Therefore, a specific analysis should be made for a specific station outside the city to be studied. The boundary of the coverage area is assumed to be treated as a circle, and the attraction radius is obtained through survey statistics or model calculations. Affected by many aspects such as neighboring stations, topography, and road network layout, the boundary of the attraction range cannot be processed in a perfectly circular manner, as shown in 


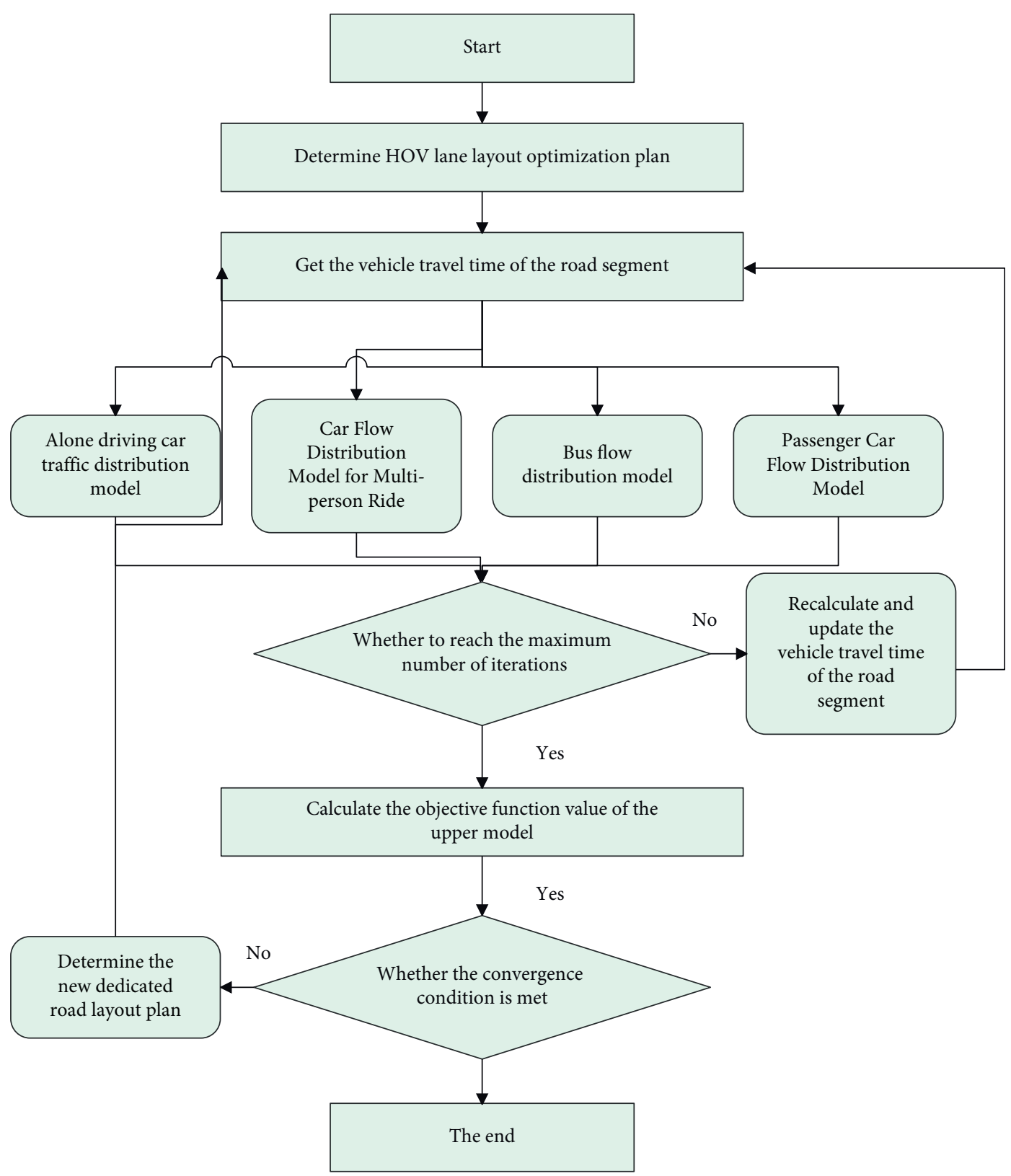

Figure 6: Model construction framework.

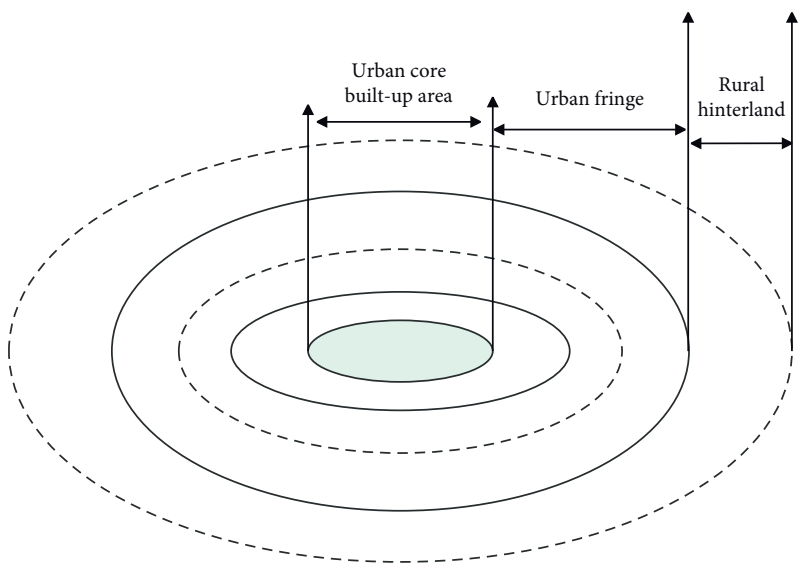

Figure 7: City structure diagram.
Figure 10. For the area where the two stations overlap, the dividing line is generally divided according to the river and road lines. This paper refers to the boundary of the attraction range of foreign " $P+R$ " park and ride stations for cars and generally adopts the form of parabola for processing, as shown in Figure 11.

The same direction HOV lane with the bus lane is set in the rightmost lane, and the HOV lane is separated from the ordinary lane by marking lines. To make it easier for passengers to get on and off the bus, parking stations are arranged on the HOV lanes, speed bumps and dashed lines that can be crossed are set $500 \mathrm{~m}$ from the front and back of the station, and solid lines that cannot be crossed are set at the rest of the road sections. The schematic diagram of road section setting is shown in Figure 12. 


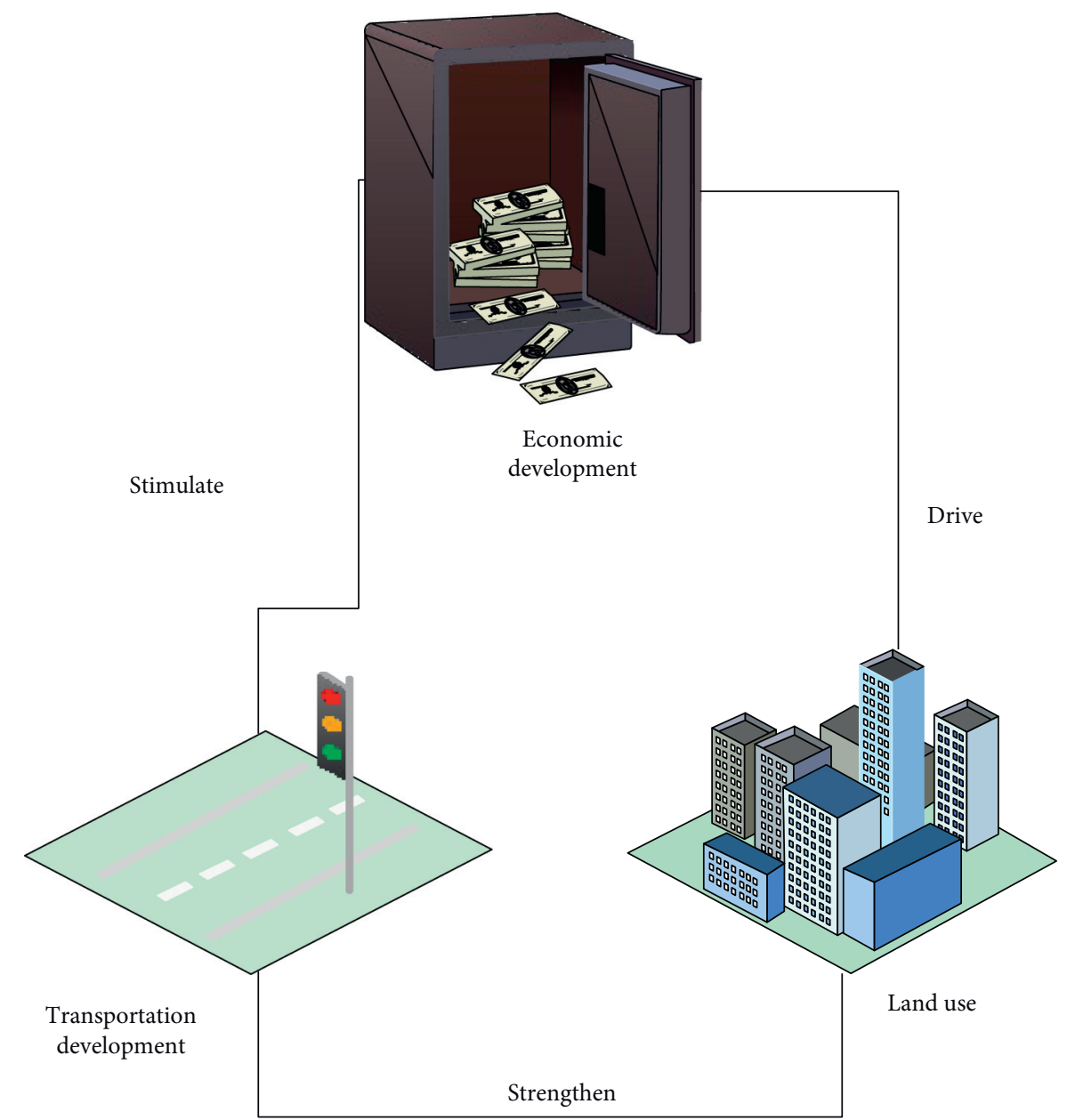

Figure 8: The relationship diagram between urban traffic, land use, and economic development.

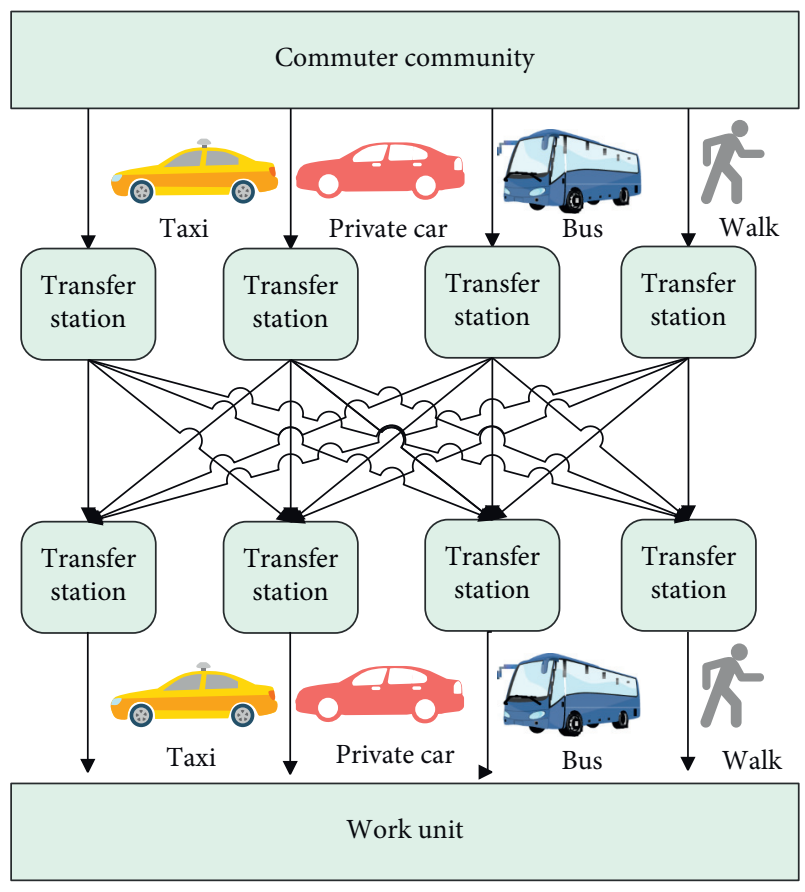

Figure 9: Schematic diagram of rail transit commuter chain.

\section{Model Test Research}

After constructing the HOV lane setting decision evaluation model, the performance of the system model is studied. The HOV model constructed in this paper is mainly used to ease urban traffic and improve urban operation efficiency. Therefore, in the process of urban operation, the system is mainly used to analyze the traffic data to determine the feasibility of the HOV lane setting, and after the feasibility is determined, the system is used to conduct the HOV lane planning analysis. In the experiment and research, this paper firstly analyzes the feasibility of HOV lane setting for the system. This paper randomly sets up 100 sets of experiments through computer simulation, collects city conditions through the network, conducts data simulation through the system, and conducts the scoring of the feasibility analysis of HOV lane setting through statistical models (quantitative analysis through subjective evaluation by experts). The results are shown in Table 1 and Figure 13. As shown in Figure 13, the abscissa is the simulation of 100 groups of HOV lanes set, and the ordinate is the feasibility score of HOV Lane set by the model.

It can be seen from the analysis results of the above charts that the system constructed in this article has a good 


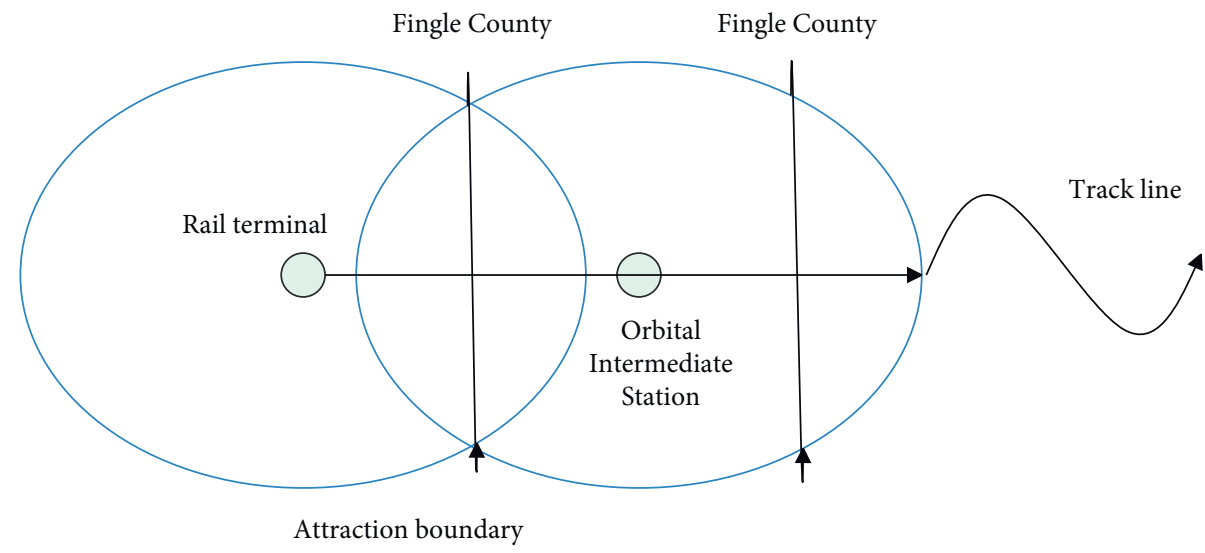

FIGURE 10: Schematic diagram of the boundary of the attraction range of a rail transit station.

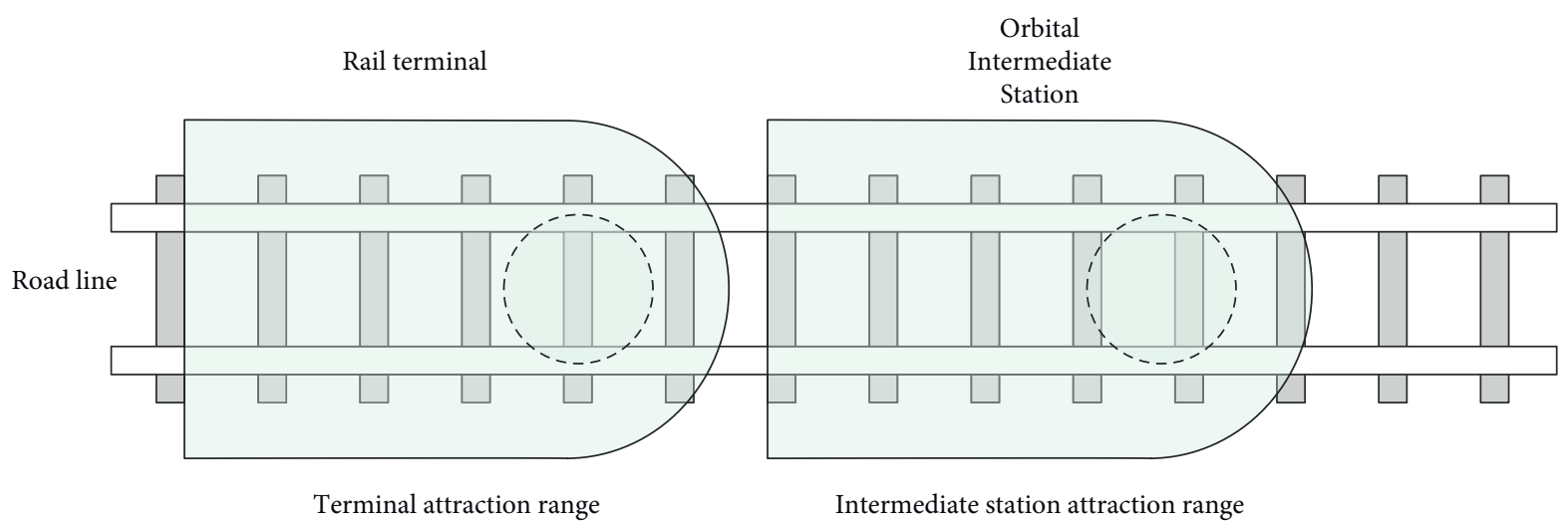

Figure 11: Schematic diagram of the parabolic boundary of the P\&R site coverage area.

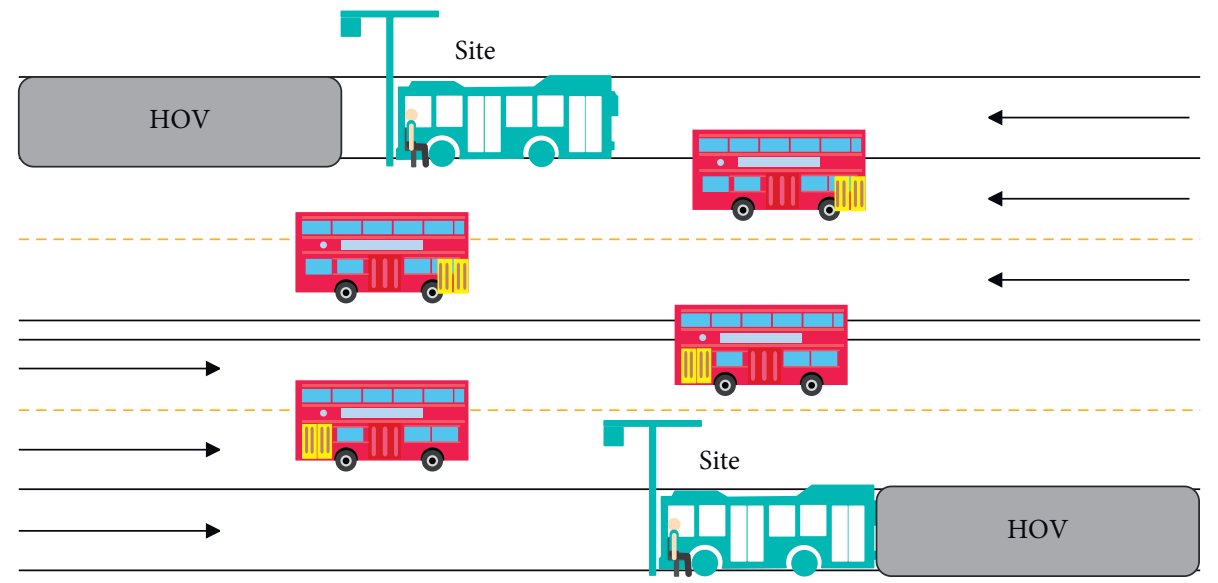

Figure 12: Schematic diagram of HOV lane road section.

effect in the feasibility analysis of HOV lane setting. Therefore, on the basis of the feasibility analysis, the HOV lane planning analysis is carried out, and the scoring method is used to count the test results (quantitative analysis through subjective evaluation by experts), as shown in Table 2 and Figure 14.

From the above test results, the decision evaluation model of HOV lane setting constructed in this paper 
TABLE 1: Statistical table of decision scores for HOV lane setting.

\begin{tabular}{|c|c|c|c|c|c|}
\hline Number & Scores & Number & Scores & Number & Scores \\
\hline 1 & 75.31 & 35 & 80.86 & 69 & 85.43 \\
\hline 2 & 76.98 & 36 & 79.47 & 70 & 86.53 \\
\hline 3 & 82.11 & 37 & 86.47 & 71 & 83.28 \\
\hline 4 & 86.24 & 38 & 78.01 & 72 & 79.87 \\
\hline 5 & 90.17 & 39 & 75.27 & 73 & 87.48 \\
\hline 6 & 89.83 & 40 & 81.47 & 74 & 80.52 \\
\hline 7 & 78.77 & 41 & 76.51 & 75 & 83.42 \\
\hline 8 & 89.68 & 42 & 86.85 & 76 & 84.11 \\
\hline 9 & 83.49 & 43 & 85.62 & 77 & 87.05 \\
\hline 10 & 84.22 & 44 & 91.18 & 78 & 76.81 \\
\hline 11 & 85.66 & 45 & 76.35 & 79 & 84.42 \\
\hline 12 & 89.05 & 46 & 78.99 & 80 & 77.12 \\
\hline 13 & 85.25 & 47 & 91.94 & 81 & 88.52 \\
\hline 14 & 79.07 & 48 & 77.09 & 82 & 90.68 \\
\hline 15 & 85.11 & 49 & 90.58 & 83 & 77.30 \\
\hline 16 & 82.68 & 50 & 88.20 & 84 & 89.64 \\
\hline 17 & 78.37 & 51 & 75.29 & 85 & 87.23 \\
\hline 18 & 81.24 & 52 & 91.19 & 86 & 80.31 \\
\hline 19 & 82.10 & 53 & 83.43 & 87 & 90.54 \\
\hline 20 & 90.70 & 54 & 75.18 & 88 & 81.66 \\
\hline 21 & 81.16 & 55 & 91.32 & 89 & 77.85 \\
\hline 22 & 91.46 & 56 & 84.59 & 90 & 79.48 \\
\hline 23 & 81.04 & 57 & 89.77 & 91 & 81.05 \\
\hline 24 & 83.05 & 58 & 84.95 & 92 & 82.81 \\
\hline 25 & 82.23 & 59 & 76.67 & 93 & 85.74 \\
\hline 26 & 77.75 & 60 & 86.56 & 94 & 85.03 \\
\hline 27 & 78.14 & 61 & 85.79 & 95 & 80.98 \\
\hline 28 & 88.56 & 62 & 89.65 & 96 & 90.45 \\
\hline 29 & 80.64 & 63 & 89.85 & 97 & 80.72 \\
\hline 30 & 78.32 & 64 & 91.67 & 98 & 80.75 \\
\hline 31 & 87.50 & 65 & 91.92 & 99 & 84.56 \\
\hline 32 & 89.87 & 66 & 84.11 & 100 & 84.64 \\
\hline 33 & 81.30 & 67 & 89.14 & & \\
\hline 34 & 83.05 & 68 & 89.43 & & \\
\hline
\end{tabular}

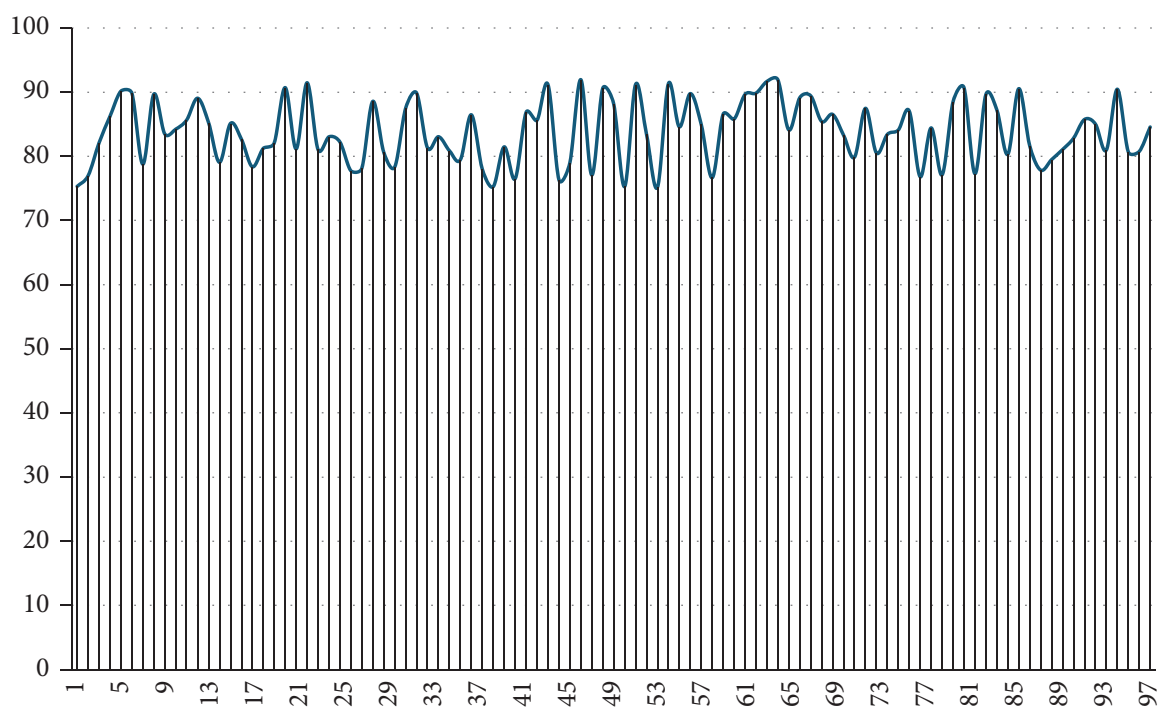

FIGURE 13: Statistical diagram of decision scores for HOV lane setting. 
TABLE 2: Statistical table of scores of HOV lane planning.

\begin{tabular}{|c|c|c|c|c|c|}
\hline Number & Scores & Number & Scores & Number & Scores \\
\hline 1 & 78.6 & 35 & 81.1 & 69 & 83.3 \\
\hline 2 & 82.6 & 36 & 83.7 & 70 & 73.2 \\
\hline 3 & 80.8 & 37 & 69.9 & 71 & 75.8 \\
\hline 4 & 84.8 & 38 & 70.8 & 72 & 74.1 \\
\hline 5 & 77.4 & 39 & 73.9 & 73 & 83.5 \\
\hline 6 & 80.2 & 40 & 76.6 & 74 & 71.6 \\
\hline 7 & 75.9 & 41 & 78.5 & 75 & 70.1 \\
\hline 8 & 73.7 & 42 & 71.0 & 76 & 69.9 \\
\hline 9 & 70.3 & 43 & 79.5 & 77 & 76.5 \\
\hline 10 & 80.3 & 44 & 73.8 & 78 & 81.8 \\
\hline 11 & 72.3 & 45 & 69.3 & 79 & 80.1 \\
\hline 12 & 77.5 & 46 & 80.3 & 80 & 73.9 \\
\hline 13 & 84.9 & 47 & 82.0 & 81 & 82.7 \\
\hline 14 & 79.0 & 48 & 71.4 & 82 & 75.2 \\
\hline 15 & 82.9 & 49 & 71.2 & 83 & 82.1 \\
\hline 16 & 82.3 & 50 & 80.0 & 84 & 73.0 \\
\hline 17 & 82.0 & 51 & 83.5 & 85 & 72.3 \\
\hline 18 & 71.9 & 52 & 75.5 & 86 & 78.3 \\
\hline 19 & 83.5 & 53 & 71.9 & 87 & 74.2 \\
\hline 20 & 82.2 & 54 & 76.0 & 88 & 84.2 \\
\hline 21 & 75.6 & 55 & 69.7 & 89 & 83.5 \\
\hline 22 & 81.0 & 56 & 83.0 & 90 & 71.9 \\
\hline 23 & 70.3 & 57 & 78.6 & 91 & 82.9 \\
\hline 24 & 70.3 & 58 & 77.9 & 92 & 70.7 \\
\hline 25 & 78.9 & 59 & 79.1 & 93 & 76.9 \\
\hline 26 & 78.6 & 60 & 75.9 & 94 & 76.5 \\
\hline 27 & 84.3 & 61 & 75.7 & 95 & 75.1 \\
\hline 28 & 83.5 & 62 & 69.9 & 96 & 73.2 \\
\hline 29 & 72.6 & 63 & 74.3 & 97 & 71.9 \\
\hline 30 & 75.9 & 64 & 84.1 & 98 & 77.6 \\
\hline 31 & 79.1 & 65 & 78.0 & 99 & 75.6 \\
\hline 32 & 78.5 & 66 & 74.8 & 100 & 76.0 \\
\hline 33 & 83.7 & 67 & 77.0 & & \\
\hline 34 & 81.5 & 68 & 75.6 & & \\
\hline
\end{tabular}

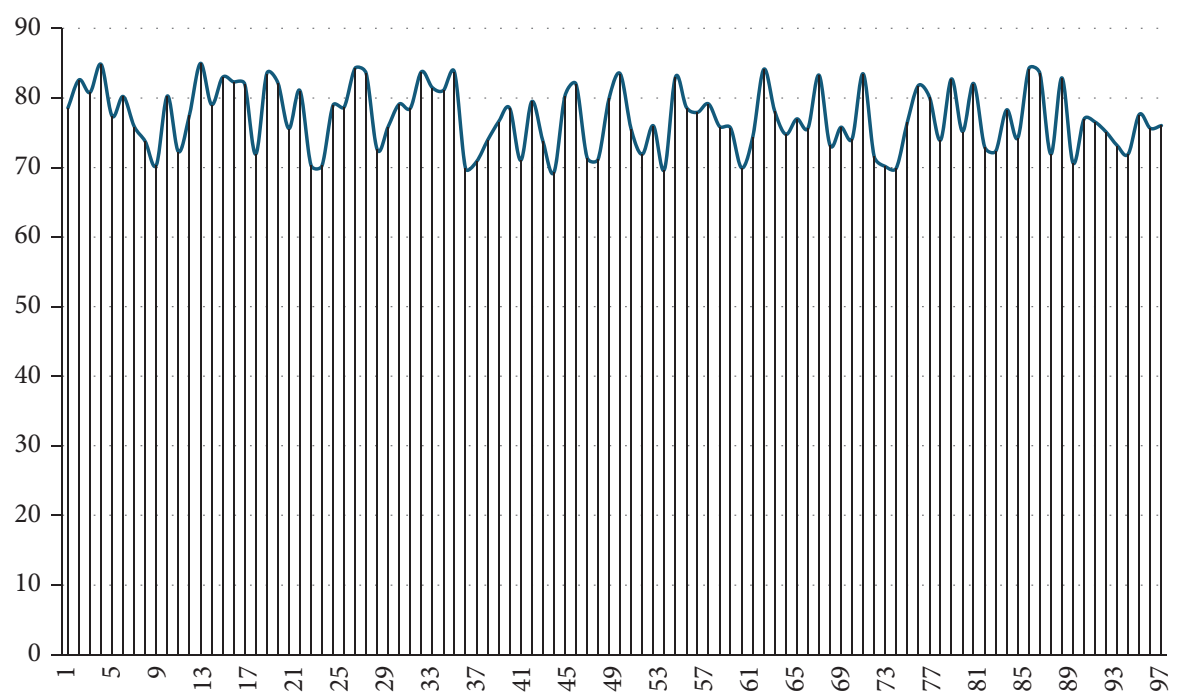

Figure 14: Statistical table of scores of HOV lane planning. 
basically meets the expected system setting requirements and has certain practical effects.

\section{Conclusion}

This paper analyzes the feasibility of setting up HOV lanes in the service rail transit commuter chain. This article first gives a theoretical introduction to the rail transit commuter chain and HOV lanes and then determines the research methods for the attraction range of rail transit stations on the periphery of the city. Moreover, this paper uses two methods of theoretical analysis and numerical analysis to illustrate the travel characteristics and ride-sharing demand of commuters within the urban rail transit attraction area and provides a method for forecasting travel demand in the HOV commuter chain. In addition, this paper determines the traffic and road conditions that should be achieved when planning and setting up HOV lanes within the attraction range of urban rail transit stations from the perspective of demand and supply. This paper combines machine learning to construct a decision-making evaluation model for HOV lane setting and studies the optimal layout model and algorithm of HOV lanes in service rail transit commuter chain. The setting, planning, and layout of HOV lanes are a two-way interactive process of traveler's path selection and designer's road planning. Finally, after the model is constructed, the performance of the system model is verified. The research results show that the route optimization model of HOV lane constructed in this paper has a certain effect. This paper analyzes the opening of HOV lane, which is of great help to reduce the flow and delay, improve the average speed and service level, and increase the number of section transportations. However, in the case of limited road resources, how to reduce the waste of no-load vehicles on road resources and tilt the right of way to multioccupant and efficient transportation mode is studied. To improve the transportation efficiency of road section, further research is needed in the future [25].

\section{Data Availability}

The experimental data used to support the findings of this study are available from the corresponding author upon request.

\section{Conflicts of Interest}

The authors declare that they have no conflicts of interest regarding this work.

\section{Acknowledgments}

This study was supported by The Fundamental Research Funds for the Central Universities: Travel Mode Transfer and Service Quality Improvement in the Area of Urban Agglomeration (300102341677); The Ministry of Education of Humanities And Social Science Project: Travel Mode Transfer Analysis And Simulation Based on Multiple Constraints (20YJC790007); and 2020 Project of The 13th Five-Year Educational Science Plan In Shaanxi Province: Research on Collaborative Teaching Reform of Large-Scale
Activity Safety Management Course Under The Background of Police Collaborative (SGH20Y1613).

\section{References}

[1] D. Nallaperuma, R. Nawaratne, T. Bandaragoda, A. Adikari, and D. Pothuhera, "Online incremental machine learning platform for big data-driven smart management," IEEE Transactions on Intelligent Transportation Systems, vol. 20, no. 12, pp. 4679-4690, 2019.

[2] M. Rath and B. Pati, "Communication improvement and traffic control based on V2I in smart city framework," International Journal of Vehicular Telematics and Infotainment Systems, vol. 2, no. 1, pp. 18-33, 2018.

[3] W.-P. Chen, A.-H. Tsai, and C.-H. Tsai, "Smart traffic offloading with mobile edge computing for disaster-resilient communication networks," Journal of Network and Systems Management, vol. 27, no. 2, pp. 463-488, 2019.

[4] "Design and implementation of smart traffic light controller using VHDL language," International Journal of Engineering \& Technology, vol. 8, no. 4, pp. 596-602, 2019.

[5] A. R. Malipatil, K. Somasundaram, M. Ashwini et al., "Smart traffic control barricade system," Journal of Critical Reviews, vol. 7, no. 14, pp. 1658-1664, 2020.

[6] A. Rehman, M. Mazhar Rathore, A. Paul, F. Saeed, and R. W. Ahmad, "Vehicular traffic optimisation and even distribution using ant colony in smart city environment," IET Intelligent Transport Systems, vol. 12, no. 7, pp. 594-601, 2018.

[7] H. K. Hamarashid, M. H. R. Saeed, and S. Saeed, "Designing a smart traffic light algorithm (HMS) based on modified round robin algorithm," Kurdistan Journal of Applied Research, vol. 2, no. 1, pp. 27-30, 2017.

[8] A. Sivanathan, H. H. Gharakheili, F. Loi, A. Rad ford, and C. Wijenayake, "Classifying IoT devices in smart environments using network traffic characteristics," IEEE Transactions on Mobile Computing, vol. 18, no. 8, pp. 1745-1759, 2018.

[9] J. D. K. H and J. Deva, "Computer vision based traffic sign sensing for smart transport," Journal of Innovative Image Processing, vol. 1, no. 1, pp. 11-19, 2019.

[10] Z. Ning, J. Huang, and X. Wang, "Vehicular fog computing: enabling real-time traffic management for smart cities," IEEE Wireless Communications, vol. 26, no. 1, pp. 87-93, 2019.

[11] J. Qiu, L. Du, D. Zhang, S. Su, and Z. Tian, "Nei-TTE: intelligent traffic time estimation based on fine-grained time derivation of road segments for smart city," IEEE Transactions on Industrial Informatics, vol. 16, no. 4, pp. 2659-2666, 2019.

[12] J. Liu, C. Zhang, and Y. Fang, "EPIC: a differential privacy framework to defend smart homes against internet traffic analysis," IEEE Internet of Things Journal, vol. 5, no. 2, pp. 1206-1217, 2018.

[13] A. Sharif, J. P. Li, and M. A. Saleem, "Internet of things enabled vehicular and ad hoc networks for smart city traffic monitoring and controlling: a review[J]," International Journal of Advanced Networking and Applications, vol. 10, no. 3, pp. 3833-3842, 2018.

[14] S. Arulselvi and M. Sundararajan, "Smart control system in traffic analysis using RTK-GPS standards," International Journal of Pure and Applied Mathematics, vol. 116, no. 15, pp. 349-353, 2017.

[15] Z. Li, R. Al Hassan, M. Shahidehpour, S. Bahramirad, and A. Khodaei, "A hierarchical framework for intelligent traffic management in smart cities," IEEE Transactions on Smart Grid, vol. 10, no. 1, pp. 691-701, 2017. 
[16] C. Raskar and S. Nema, "Modified fuzzy-based smart barricade movement for traffic management system," Wireless Personal Communications, vol. 116, no. 4, pp. 3351-3370, 2021.

[17] L. Sumi and V. Ranga, "Intelligent traffic management system for prioritizing emergency vehicles in a smart city," International Journal of Engineering, vol. 31, no. 2, pp. 278-283, 2018.

[18] A. I. Adekitan, J. Abolade, and O. Shobayo, "Data mining approach for predicting the daily Internet data traffic of a smart university," Journal of Big Data, vol. 6, no. 1, pp. 1-23, 2019.

[19] M. Li, P. Si, and Y. Zhang, "Delay-tolerant data traffic to software-defined vehicular networks with mobile edge computing in smart city," IEEE Transactions on Vehicular Technology, vol. 67, no. 10, pp. 9073-9086, 2018.

[20] P. Bellucci and E. Cipriani, "Data accuracy on automatic traffic counting: the SMART project results," European transport research review, vol. 2, no. 4, pp. 175-187, 2010.

[21] X. Wang and Z. Li, "Traffic and transportation smart with cloud computing on big data," Indian Journal of Chemistry, Section A, vol. 13, no. 1, pp. 1-16, 2016.

[22] G. Hou, S. Chen, Y. Zhou, and J. Wu, "Framework of microscopic traffic flow simulation on highway infrastructure system under hazardous driving conditions," Sustainable and Resilient Infrastructure, vol. 2, no. 3, pp. 136-152, 2017.

[23] Y. Xue, Y. Zhang, D. Fan, P. Zhang, and H.-d. He, "An extended macroscopic model for traffic flow on curved road and its numerical simulation," Nonlinear Dynamics, vol. 95, no. 4, pp. 3295-3307, 2019.

[24] G. Ghermandi, S. Fabbi, A. Bigi et al., "Impact assessment of vehicular exhaust emissions by microscale simulation using automatic traffic flow measurements," Atmospheric Pollution Research, vol. 10, no. 5, pp. 1473-1481, 2019.

[25] V. O. Matthews, S. I. Uzairue, and E. Noma-Osaghae, "Design and simulation of a smart traffic system in a campus community," Journal of Emerging Technologies and Innovative Research (JETIR), vol. 5, no. 7, pp. 492-497, 2018. 